\title{
Mental health system change
}

\section{Bridging hospital and community mental health care}

\author{
Meagan Wiederman, Celina Everling, Nathan Leili, Saba Shahab
}

\begin{abstract}
Mental health is defined as a state of well-being in which individuals realize their potential, cope with normal stress, and work effectively. Due to transitions of mental health care from psychiatric institutes to hospital- and community-based care, treatment of mental illness has suffered from fragmentation and poor connectivity between hospital care providers and community support programs. It is essential that the hospital and community are connected in order for patients to feel supported by their community at all stages of their recovery. Patients report that they need to feel like they are supported in the community to have hope for continued improvement. In order to tackle the problem of connecting the hospital and community to promote the recovery of people with severe mental illness, a team of four students, Re-MIND London, proposed an innovative solution suite. This involved creating a liaison between community and hospital, crafting a shared menu of resources, distributing information through education and samplers, having patients engage in community outings, and providing peer mentor support. In implementing this solution, we have helped rekindle connection between Parkwood Institute Mental Health Care and the Canadian Mental Health Association in London, ON at an administrative, staff, and patient level. In implementing parts of this innovative solution, our team learned self-empowerment to challenge the existing system. We are connecting individuals with mental illness and organizations to form strong and sustainable partnerships to help to reintegrate them into the community throughout treatment and recovery.
\end{abstract}

\section{INTRODUCTION}

Mental health is a significant, prevalent issue

The World Health Organization defines mental health as a state of well-being in which "every individual realizes [their] own potential, can cope with the normal stresses of life, [and] can work productively and fruitfully."1 Mental health is as important as physical health; both contribute to a person's quality of life. Mental illness is prevalent and widespread: 1 in 5 people will have a mental health condition during any given year, and $50 \%$ of people will have a mental health condition at some point in their life. ${ }^{2}$ Globally, the economic costs of mental illness were estimated at US\$2.5 trillion. ${ }^{3}$ More than $70 \%$ of people with mental health conditions worldwide do not receive any mental health care treatment. ${ }^{4}$ This may be a global lack of awareness of and knowledge about mental illness which perpetuates stigma and discrimination, ${ }^{4-7}$ leading to exclusion of individuals with mental illness from meaningful participation in their communities. Effective systems should be developed for recovery for persons with mental illness.

Mental health care problems locally: London, $\mathrm{ON}$

Western countries like Canada experienced significant changes to the way mental illnesses are treated when psychiatric institutes were assimilated into current hospital-based care model. ${ }^{2}$ Prior to the psychiatric reform of the 1970s, individuals with mental illnesses were exclusively treated in mental health institutions. The reform moved individuals with mental illness into general hospitals; however, it failed to fully integrate mental health care into general hospitals and created a deficit of hospital to community transition services. A meta-analysis by Vázquez-Bourgon et al in 2012 suggests that this incomplete transition leaves most Western countries with insufficient and fragmented community services, where the acute care of severe mentally ill patients rests solely with traditional inpatient services. ${ }^{8}$

The mental health care system in London and Middlesex county is complicated to navigate, as there is no centralized point to access information for all available mental health services in this region. Two of the many leaders in mental health care in London and Middlesex county are St. Joseph's Health Care London Parkwood Institute Mental Health Care (Parkwood), which provides care to people with severe mental illness longterm stays in hospital, and the Canadian Mental Health Association Middlesex (CMHA) which offers community-based mental health services for adults with mental illness. In London's current system, hospitalized individuals may have limited access to supports within their communities. Many individuals with severe mental illness experience difficulties with activities of daily living and are at risk of missing physician visits, discontinuing medications, and experiencing suicidal thoughts immediately post-discharge from hospital. ${ }^{9}$ As a result, post-discharge is a crucial window for intervention aimed at helping these individuals cope and integrate into their communities. When discharged, hospital supports are removed. The sudden removal of hospital supports is difficult for these individuals, particularly those with no transitional care, ${ }^{10}$ and can lead to a cycle of readmission. ${ }^{11}$ This raises a complex problem: "How can we connect people with severe mental illness to supports in the London community in a way that promotes their recovery?"

\section{METHODS}

People can develop support networks and engage in activities that are meaningful to them through the connection of hospital and community-based resources, which ultimately enhances their journey of recovery. The authors, Re-MIND London, partnered 
with CMHA and Parkwood to pilot a solution to the proposed question and connect the hospital and community. We searched the literature to find, then pitch, implement, and refine solutions to the proposed problems and challenges of Parkwood and CMHA. Feedback and comments from patients and staff were accepted and recorded at an open booth in the Parkwood lobby.

\section{RESULTS}

Innovating the mental health care system locally

The main challenge of this problem was coordinating the various stakeholders, and the diverse and unique journey of recovery for every patient. This means that any solution addressing connections between the hospital and community needs to be robust and flexible in order to be useful to patients. To deliver an impactful and personalizable solution, Re-MIND London proposed the following solutions:

- Involve a community-knowledge representative with the inpatient client team, to work with the patient at the hospital to make community services a part of treatment.

- Compile a single, online menu of all mental health resources to make navigating the system easy and accessible, allowing patients to explore their options and tailor their treatment plans.

- Distribute community resource information, such as pamphlets, in the hospital to inform patients and hospital staff of community services.

- Demonstrate community programming "samplers" in the hospital to reduce the barriers of learning about a service, meet new health care providers and other patients, and do new activities.

- Take hospital patients on outings to community programs to see where programs are held, meet the community members that attend, and begin a route of using community services to promote comfort with community resource use.

- Connect community peer support mentors with lived experience of mental illness with inpatients to provide opportunities to share their story, socialize, and learn from another person's mental health journey.

A common theme in successful transitional planning models is individualized, needs-based support through community services in collaboration with the hospital. ${ }^{12,13}$ Our suite of solutions aims to connect the hospital and community in an integrated model, as inspired by the $50 \%$ reduction in readmission rates after an integrated care project in Germany. ${ }^{14}$ A "bridging program" was shown to be effective in connecting a multidisciplinary clinical team for improved patient sense of wellbeing, decreased symptom intensity, and improved access and engagement with community resources. ${ }^{11}$ This evidence supports our suggested liaison, shared menus, and information sessions. Our solution proposes to use a peer-delivered transitional support program, which has previously been shown to increase functional recovery by up to $10 \%$, improve psychological wellbeing, and reduce readmission rates. ${ }^{15,16}$

\section{Progress}

Thus far, we have rekindled a strong partnership amongst the relevant administration. Meetings between Christine Samson (CMHA, Director of Intensive Case Management and Clinical Services) and Deb Gibson (Parkwood, Director) have resulted in a plan for future installment of the remaining proposed solutions. Distributing CMHA resources in Parkwood has already shown improvement in patient and physician knowledge of community services. Patients who learn about what services they can continue to use in the community are reporting interest in many community programs, such as smoking cessation, anxiety/depression/bipolar groups, and crisis phone services. Patients have stated that knowing what mental health services are available in the community has made them "feel like the [hospital] is still in [the London] community." This sense of connection between the hospital with the community is the main goal of the project. Additionally, it is important to educate hospital staff to promote continued community engagement with their patients. CHMA has invited Parkwood staff for guided tours of their crisis center, allowing Parkwood staff to confidently recommend their services. Staff have reported that this "[helps with] a plan for some time [after discharge from Parkwood]."

\section{CONCLUSION}

Significance of the project

This collaboration innovation will continue to foster a deep network of connection between the community and hospital, which is essential for improving the mental health system. In sustainably continuing this project, the administration of Parkwood and CMHA are enthusiastic and dedicated to integrating collaboration into their practice. Mental illness can be socially isolating and personally straining. It is essential to offer individuals with mental illness support networks and care systems for their personalized recovery journey. Parkwood and CMHA collaborating on care will integrate the hospital within the community and support individuals' personal recovery.

\section{A reflection on changing the mental health care system}

As a group of students working on this project to improve the mental health care system, we are smaller than these organizations. While we originally felt insignificant proposing changes to these organizations, through connecting these two mental health leaders - Parkwood and CMHA - we have come to recognize that leaders will acknowledge ideas that challenge their system for growth. Reflecting on our progress helps our team reconsider alternative solutions to this problem. Our team has discovered that in order to achieve sustainability, the initiative must leverage the strengths of both partners. Interventions with multiple partners must strategize for equitable contribution to funding and resource allocation. Collaboration between all partners is critical to truly foster connection in mental health care for the multidisciplinary care for patients' personal recovery. For example, early in our problem search, we discovered that there were misunderstandings about CMHA programming cycling seasonally, and that distributing an updated program guide each season would be essential to inform Parkwood patients about current services and to prevent Parkwood staff from making referrals to programs that had already finished. Parkwood offered to sponsor the printing costs of CMHA program 
guides for distribution within its floors while CMHA designated a staff member to constantly update Parkwood about changes to the program. This problem solving and collaboration helped to better inform Parkwood patients about CMHA programming and reassured Parkwood staff about recommending CMHA services. Before this project, the organizations expressed confusion about the other's care model. When collaborating with the two organizations, we quickly noticed and bridged this confusion. Therefore, collaboration between all partners is critical to truly foster connection in mental health care for the multidisciplinary care for patients' personal recovery.

\section{REFERENCES}

1. World Health Organization. Universal health coverage (UHC). 2018 Oct 18. [cited 2019 Aug 4] Available from: http://www.who.int/newsroom/fact-sheets/detail/universal-health-coverage-(uhc)

2. Mental Health Commission of Canada. Making the case for investing in mental health in Canada [Internet]. Mental Health Commission of Canada; 2013 [cited 2019 Aug 4]. p. 1-28. Available from: https://www. mentalhealthcommission.ca/sites/default/files/2016-06/Investing_in Mental_Health_FINAL_Version_ENG.pdf

3. Trautmann S, Rehm J, Wittchen HU. The economic costs of mental disorders: Do our societies react appropriately to the burden of mental disorders? EMBO Rep. 2016 Sept;17(9):e201642951. https://doi. org/10.15252/embr.201642951

4. Henderson C, Evans-Lacko S, Thornicroft G. Mental illness stigma, help seeking, and public health programs. Am J Public Health. 2013 May;103(5):777-80. https://doi.org/10.2105/AJPH.2012.301056

5. Wainberg ML, Scorza P, Shultz JM, et al. Challenges and opportunities in global mental health: a research-to-practice perspective. Curr Psychiatry Rep. 2017 May;19(5):28. https://doi.org/10.1007/s11920-017$\underline{0780-\mathrm{Z}}$

6. Patel V. Mental health in low- and middle-income countries. Br Med Bull. 2007;81-82(1):81-96. https://doi.org/10.1093/bmb/ldm010

7. Kopinak JK. Mental health in developing countries: challenges and opportunities in introducing western mental health system in Uganda. Int J MCH AIDS. 2015;3(1):22-30.

8. Vázquez-Bourgon J, Salvador-Carulla L, Vázquez-Barquero JL. Community alternatives to acute inpatient care for severe psychiatric patients. Actas Esp Psiquiatr 2012;40(5):323-32.

9. Roby RC, Shapira Goldstein RL, Rose ES, et al. Post-discharge experiences of people with psychosis following partial hospital program completion. Occup Ther Ment Health. 2018;34(3):272-84. https://doi.org/10.1080/0164212X.2017.1418698

10. Niimura J, Tanoue M, Nakanishi M. Challenges following discharge from acute psychiatric inpatient care in Japan: patients' perspectives. Journal of psychiatric and mental health nursing. 2016 Nov-Dec;23(910):576-84. https://doi.org/10.1111/jpm.12341

11. Madi N, Zhao H, Li JF. CIHI survey: hospital readmissions for patients with mental illness in Canada. Healthc Q. $2007 \mathrm{Feb} ; 10(2): 30-2$. https:// doi.org/10.12927/hcq.2007.18818

12. Chong WW, Aslani P, Chen TF. Shared decision-making and interprofessional collaboration in mental healthcare: a qualitative study exploring perceptions of barriers and facilitators. J Interprof care. 2013;27(5):373-9. https://doi.org/10.3109/13561820.2013.785503
13. Scanlan JN, Hancock N, Honey A. Evaluation of a peer-delivered, transitional and post-discharge support program following psychiatric hospitalisation. BMC Psychiatry. 2017 Aug;17:307. https://doi. org/10.1186/s12888-017-1469-x

14. Schmidt-Kraepelin C, Janssen B, Gaebel W. Prevention of rehospitalization in schizophrenia: results of an integrated care project in Germany. Eur Arch Psychiatry Clin Neurosci. 2009 Oct;259(2):205. https://doi.org/10.1007/s00406-009-0056-7

15. Steffen S, Kösters M, Becker T, et al. Discharge planning in mental health care: a systematic review of the recent literature. Acta Psychiatr Scand. 2009 Jul;120(1):1-9. https://doi.org/10.1111/j.16000447.2009.01373.x

16. Vigod SN, Kurdyak PA, Dennis CL, et al. Transitional interventions to reduce early psychiatric readmissions in adults: systematic review. 2013. In: Database of Abstracts of Reviews of Effects (DARE): Qualityassessed Reviews [Internet]. York (UK): Centre for Reviews and Dissemination (UK); 1995- [cited 2019 Aug 4]. Available from: https:// www.ncbi.nlm.nih.gov/books/NBK132331/ 